\title{
Efeitos da Energia da Dieta sobre Desempenho, Rendimento de Carcaça e Gordura Abdominal de Frangos de Corte
}

\section{Ariel Antônio Mendes ${ }^{1}$, Joerley Moreira ${ }^{2}$, Edson Gonçalves de Oliveira ${ }^{3}$, Edivaldo Antônio Garcia $^{1}$, Marina Isabel Mateus de Almeida ${ }^{3}$, Rodrigo Garófallo Garcia ${ }^{4}$}

RESUMO - Um experimento foi realizado com o objetivo de avaliar o efeito do nível de energia da dieta sobre desempenho, rendimento de carcaça e porcentagem de gordura abdominal de frangos de corte. Foram utilizados 2400 pintos de um dia da linhagem Ross 308, criados em boxes até 42 dias de idade. O delineamento experimental adotado foi o inteiramente casualizado em esquema fatorial 6 x 2 (energia x sexo). Os tratamentos consistiram de combinações de seis níveis de energia (2.900, 2.960, 3.020, 3.080, 3.140 e $3.200 \mathrm{kcal} \mathrm{EM/kg)} \mathrm{e} \mathrm{dos} \mathrm{sexos,} \mathrm{com} \mathrm{quatro} \mathrm{repetições} \mathrm{de} 50$ aves cada. No período de 1 a 42 dias de idade, houve redução no consumo de ração e melhora na conversão alimentar à medida que se aumentou o nível de energia da ração. Os machos apresentaram melhores resultados de desempenho que as fêmeas, exceto para mortalidade. À medida que se acrescentou energia na dieta, houve efeito linear na porcentagem de gordura abdominal e no rendimento de asas, mas não houve efeito sobre o rendimento de carcaça e das demais partes. Os machos apresentaram maiores porcentagens de pernas e de carne de pernas e menores de carne de peito e de gordura abdominal que as fêmeas.

Palavras-chave: desempenho, energia, frangos de corte, rendimento de carcaça

\section{Effect of Dietary Energy on Performance, Carcass Yield and Abdominal Fat of Broiler Chickens}

\begin{abstract}
An experiment was conducted to evaluate the effect of dietary energy level on performance, carcass yield and abdominal fat of broiler chickens. Two thousand and four hundred day-old Ross 308 chicks were raised until 42 days of age in floor pens. The experimental design was a completly randomized in a 6 x 2 factorial design (energy x sex). The treatment was consisted of combinated levels of energy: 2900, 2960, 3020, 3080, 3140 and 3200 kcal/kg ME and sex, with four replicates of 50 birds. From 1 to 42 days of age, feed consumption and feed conversion decreased as energy level increased. Males presented the best performance results than females, except for mortality. There was a linear effect of energy level on percentage abdominal fat and wings, but there was no effect on carcass yield and on all other parts of carcass. Males presented higher total legs and leg meat percentages and lower breast meat and abdominal fat than females.
\end{abstract}

Key Words: broilers, carcass yield, dietary energy, performance

\section{Introdução}

No passado, o principal objetivo da produção avícola consistia na obtenção de ótimo peso ao abate, associado à melhor conversão alimentar. Atualmente, existem, além dos citados, outros critérios importantes, como rendimento de carcaça, produção de carne de peito e de pernas e qualidade da carcaça. A importância dessas características varia de empresa para empresa e depende do tipo de produto comercializado. Com a introdução de linhagens de alto rendimento no mercado brasileiro, o setor adotou novos critérios de manejo e nutrição dos frangos de corte, a fim de maximizar a produtividade e otimizar os custos. Com isso, a definição de níveis ótimos de energia da dieta passou a ser fundamental, uma vez que os frangos das linhagens atuais têm exigências nutricionais específicas e são diferentes dos frangos de alguns anos atrás.

O nível energético das rações interfere substancialmente no resultado de desempenho de frangos de corte. Segundo Bastos et al. (1998), Albuquerque et al. (2000), Luchesi (2000), Rosa et al. (2000), Silva et al. (2000), Moreira et al. (2001) e Watanabe et al.

\footnotetext{
${ }^{1}$ Professores do DPEA da FMVZ/UNESP, campus de Botucatu - SP (arielmendes@fca.unesp.br; egarcia@fca.unesp.br).

2 Pós-Doutorando em Zootecnia pelo DPEA da FMVZ-UNESP, campus de Botucatu-SP. Bolsista do CNPq. E.mail: joerley@fca.unesp.br 3 Professores do Departamento de Genética da Universidade Federal do Paraná, Curitiba-PR. E.mail: ego@ufpr.br

${ }^{4}$ Doutorando em Genética pelo DPEA da FMVZ/UNESP, campus de Botucatu - SP. Bolsista da FAPESP. E.mail: garofallo@fca.unesp.br
} 
(2001), o acréscimo no nível de energia das rações proprociona melhor ganho de peso e conversão alimentar, porém acarreta aumento no teor de gordura abdominal. Com relação ao rendimento de carcaça e cortes, vários autores verificaram efeito significativo ao modificarem os níveis de energia da dieta (Lushesi, 2000; Rosa et al., 2000; Mendes et al., 2001; Moreira et al., 2001), ao passo que outros autores não verificaram o mesmo efeito (Zanusso et al., 1999; Oliveira Neto et al., 2000; Leandro et al., 2000), o que pode ser explicado por diferenças de manejo, épocas de criação, linhagens e densidade populacionais e, evidentemente pela diferença entre os níveis de energia utilizados, entre outros fatores.

No presente trabalho, objetivou-se reavaliar o efeito de diferentes níveis de energia da dieta sobre o desempenho, o rendimento de carcaça e a gordura abdominal de frangos de corte, com intuito de verificar se os conceitos amplamente aceitos sobre energia na dieta, adotados no passado, persistem nas linhagens atuais.

\section{Material e Métodos}

O experimento foi conduzido nas instalações experimentais do setor de avicultura da Faculdade de Medicina Veterinária e Zootecnia da UNESPCampus de Botucatu, SP, no período de 3 de novembro a 15 de dezembro de 1999. Foram utilizados 2.400 pintos de corte de um dia de idade, da linhagem Ross 308, sexados e distribuídos em 48 boxes de 5,0 $\mathrm{m}^{2}$ cada. As aves foram submetidas a um delineamento inteiramente casualizado com arranjo fatorial $6 \times 2$, ou seja, seis níveis de energia (2.900, 2.960, 3.020, 3.080, 3.140 e $3.200 \mathrm{kcal} \mathrm{EM/kg)} \mathrm{e} \mathrm{dois} \mathrm{sexos,} \mathrm{com}$ quatro repetições de 50 aves cada.

Foi utilizado um programa de alimentação em duas fases: inicial (de 1 a 21 dias) e de crescimento/ final (de 21 a 42 dias). As rações experimentais foram idênticas, variando apenas quanto aos níveis de energia, de acordo com os tratamentos experimentais, sendo adotados os níveis utilizados pela indústria do Estado de São Paulo (Tabela 1).

O desempenho das aves foi avaliado segundo o peso final, o ganho de peso, o consumo de ração, a conversão alimentar e a mortalidade por fase e no período total. Aos 42 dias de idade, foram amostradas quatro aves por parcela, com 48 aves por tratamento e 192 no total, para determinação do rendimento de carcaça, de cortes e de gordura abdominal. As aves foram abatidas após jejum de oito horas no abatedouro experimental de aves da FMVZ, distante $3 \mathrm{~km}$ da granja. O rendimento de carcaça foi calculado em relação ao peso vivo antes do abate [\%rendimento de carcaça $=($ peso carcaça $* 100 /$ peso vivo $)]$ e o rendimento das partes, em função do peso da carcaça eviscerada, sem penas, mas com pés, cabeça e pescoço [\%rendimento de partes $=$ (peso da parte *100/peso carcaça]. Foram avaliados os seguintes parâmetros: peso vivo, carcaça, pernas, carne de pernas, osso de pernas, pele de pernas, peito total, carne do peito, osso do peito, pele do peito, asas, dorso e porcentagem de gordura abdominal.

A análise estatística dos resultados foi realizada utilizando-se o pacote estatístico SAEG, segundo Euclydes (1983).

\section{Resultados e Discussão}

As temperaturas máximas e mínimas verificadas no interior do galpão experimental durante o experimento foram de $31,1^{\circ} \mathrm{C} \pm 3,6^{\circ} \mathrm{C}$ e $18,9^{\circ} \mathrm{C} \pm 1,9^{\circ} \mathrm{C}$, respectivamente.

Os resultados de desempenho encontram-se na Tabela 2. No período de 1 a 21 dias de idade, o sexo das aves influenciou os parâmetros avaliados, exceto a mortalidade, sendo que os machos apresentaram desempenho superior às fêmeas em todos os parâmetros. Não foi encontrada interação significativa $(p>0,05)$ entre nível de energia e sexo. Os níveis de energia avaliados também influenciaram o desempenho das aves aos 21 dias, com exceção da mortalidade. O peso vivo e o ganho de peso apresentaram efeito quadrático $(\mathrm{p}<0,01)$, comprovando que a elevação do nível de energia na dieta promoveu aumentos nestes parâmetros, o que pode ser verificado até o nível de $3.140 \mathrm{kcal} \mathrm{EM/kg}$, no entanto, houve redução ao utilizar o nível de $3.200 \mathrm{kcal} \mathrm{EM} / \mathrm{kg}$. Estes resultados para peso vivo e para ganho de peso até os 21 dias indicam que, nesta fase, o uso de níveis acima de $3.140 \mathrm{kcal}$ EM/kg não deve ser recomendado, uma vez que promovem redução do desempenho, provavelmente em função da queda no consumo de ração, que apresentou efeito linear negativo $(\mathrm{p}<0,01)$, 


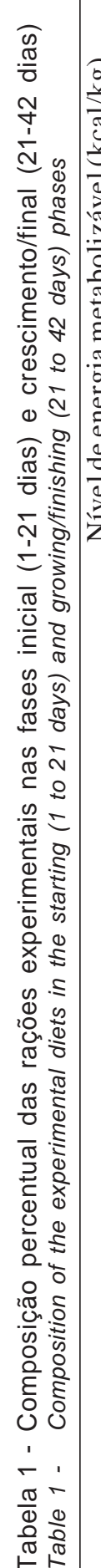

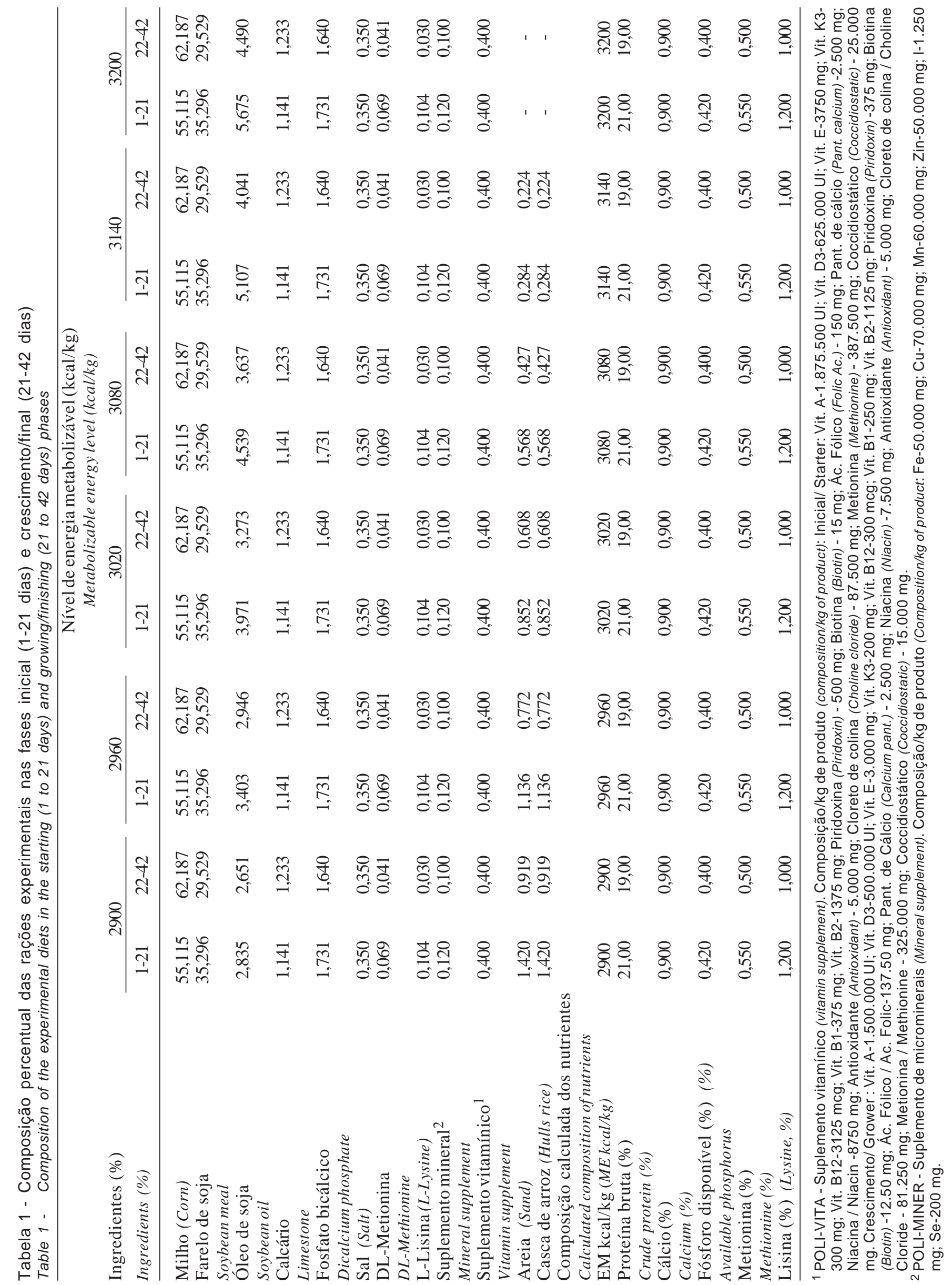

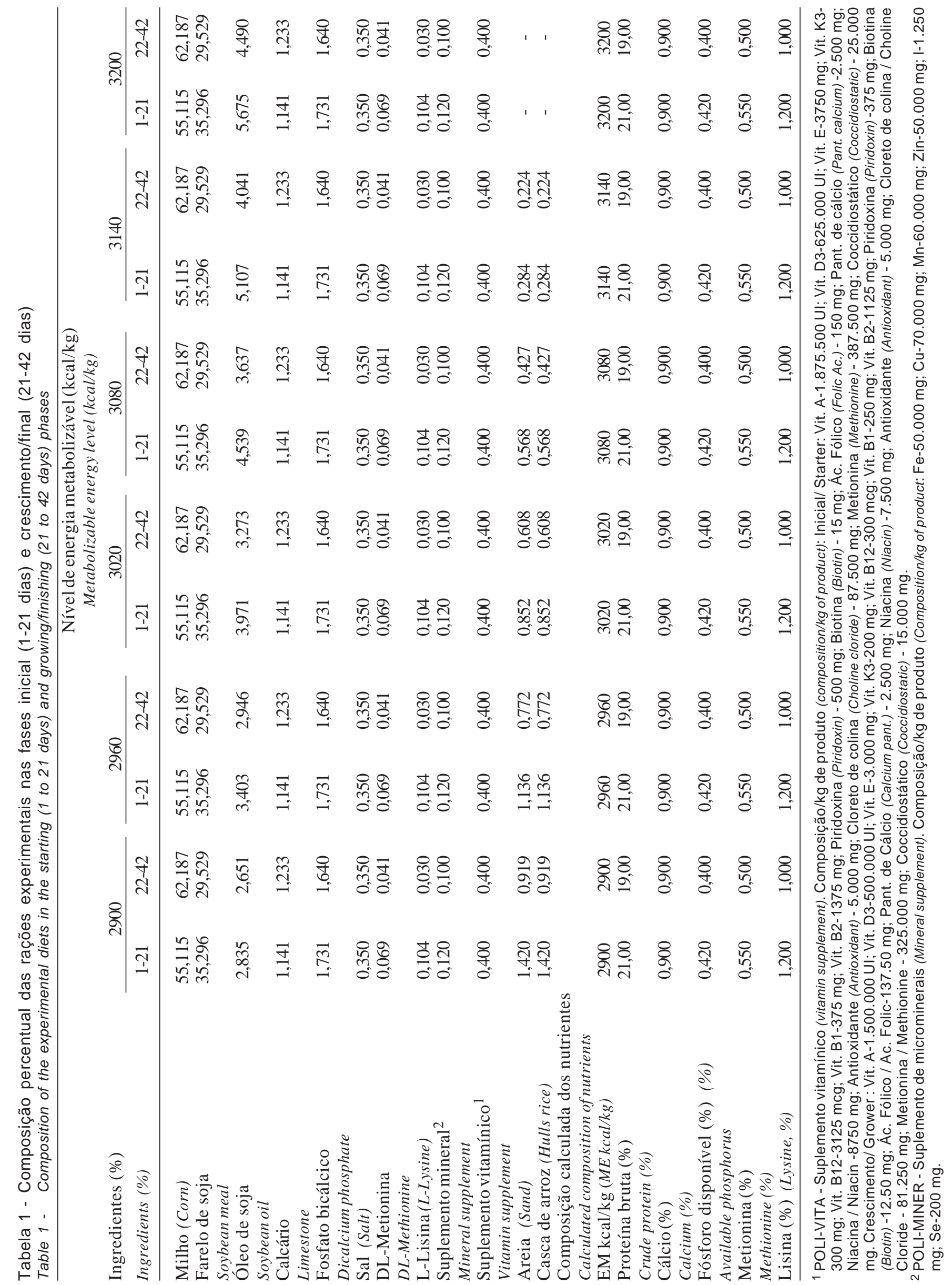

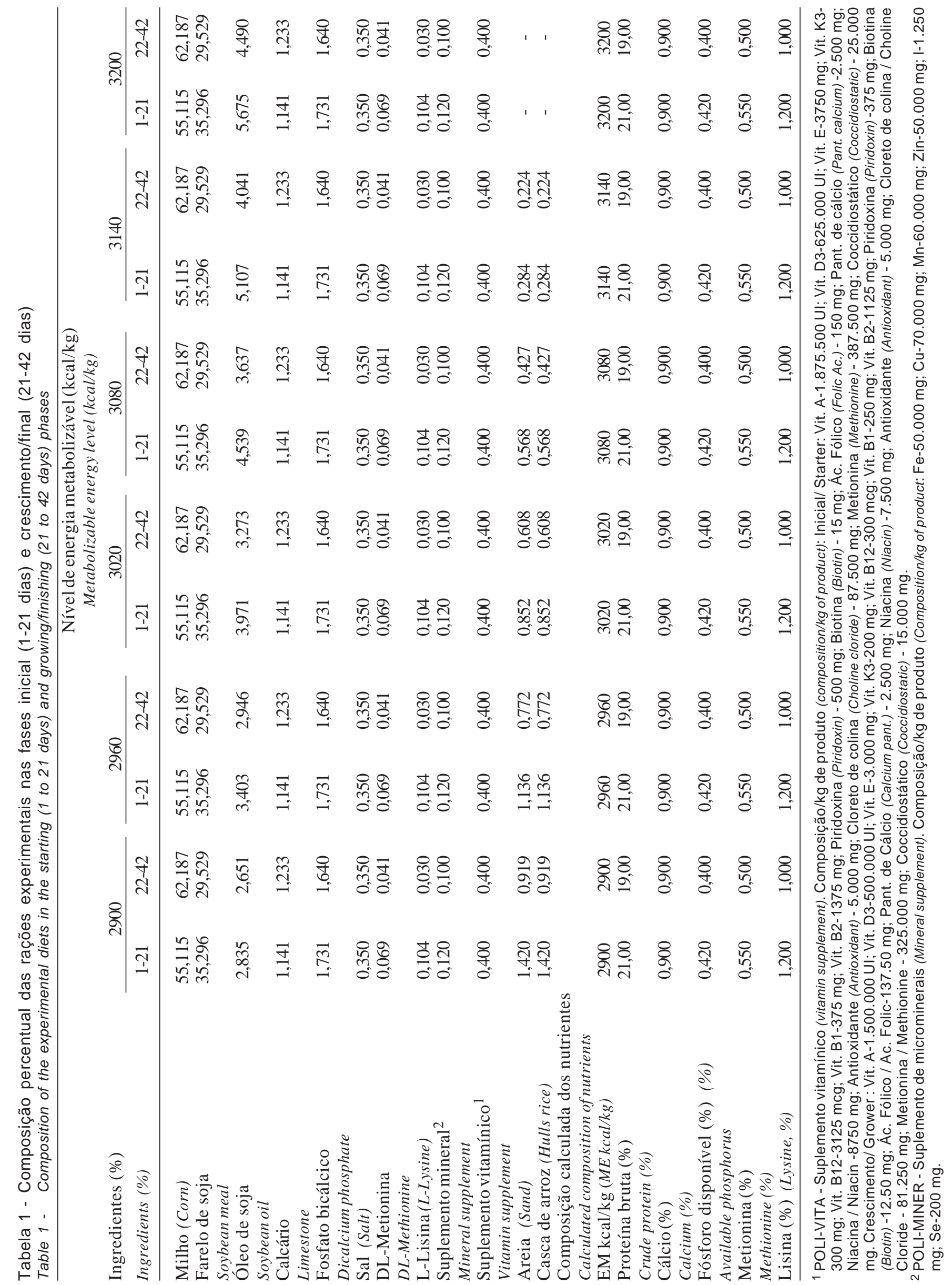

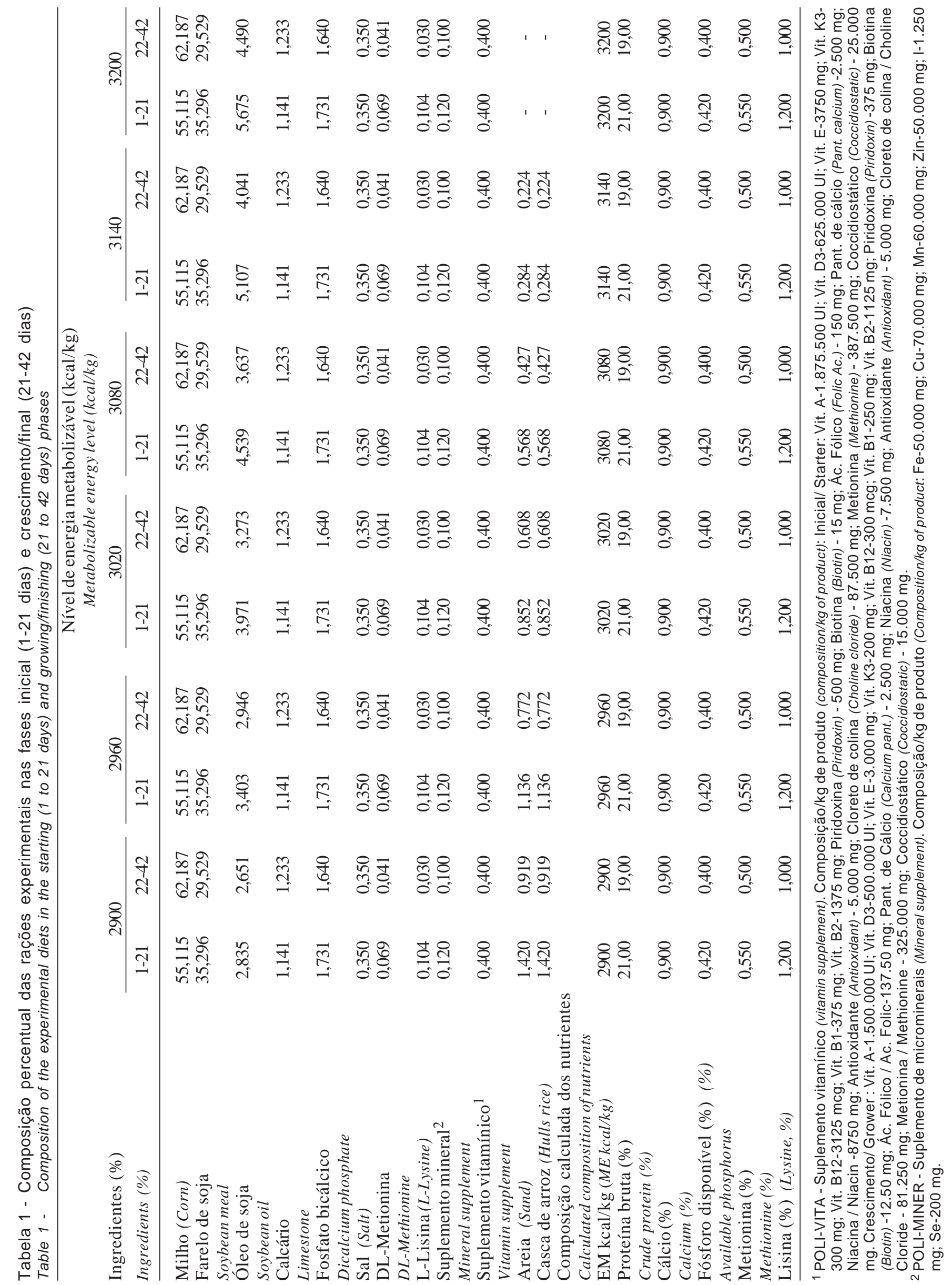

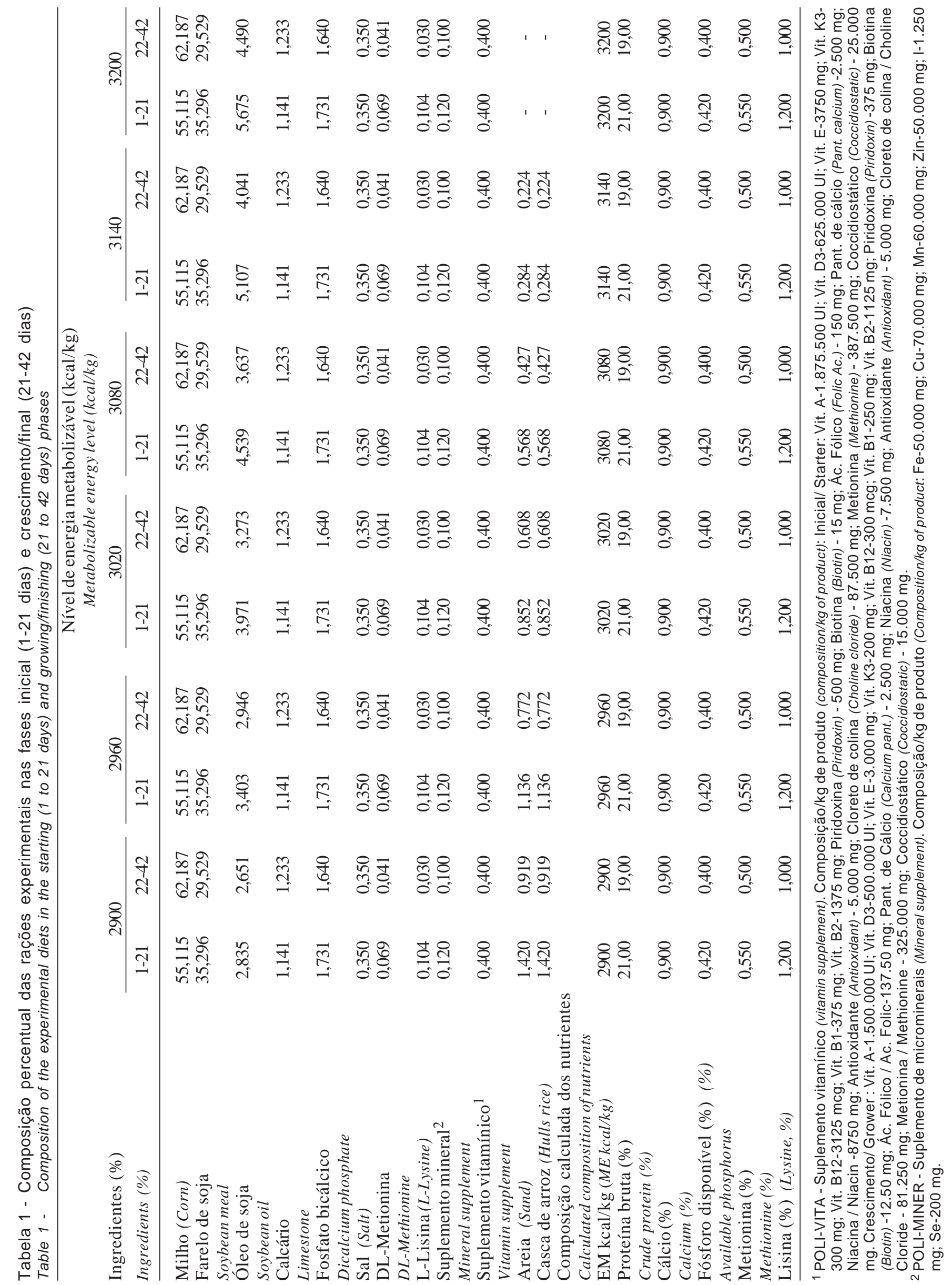

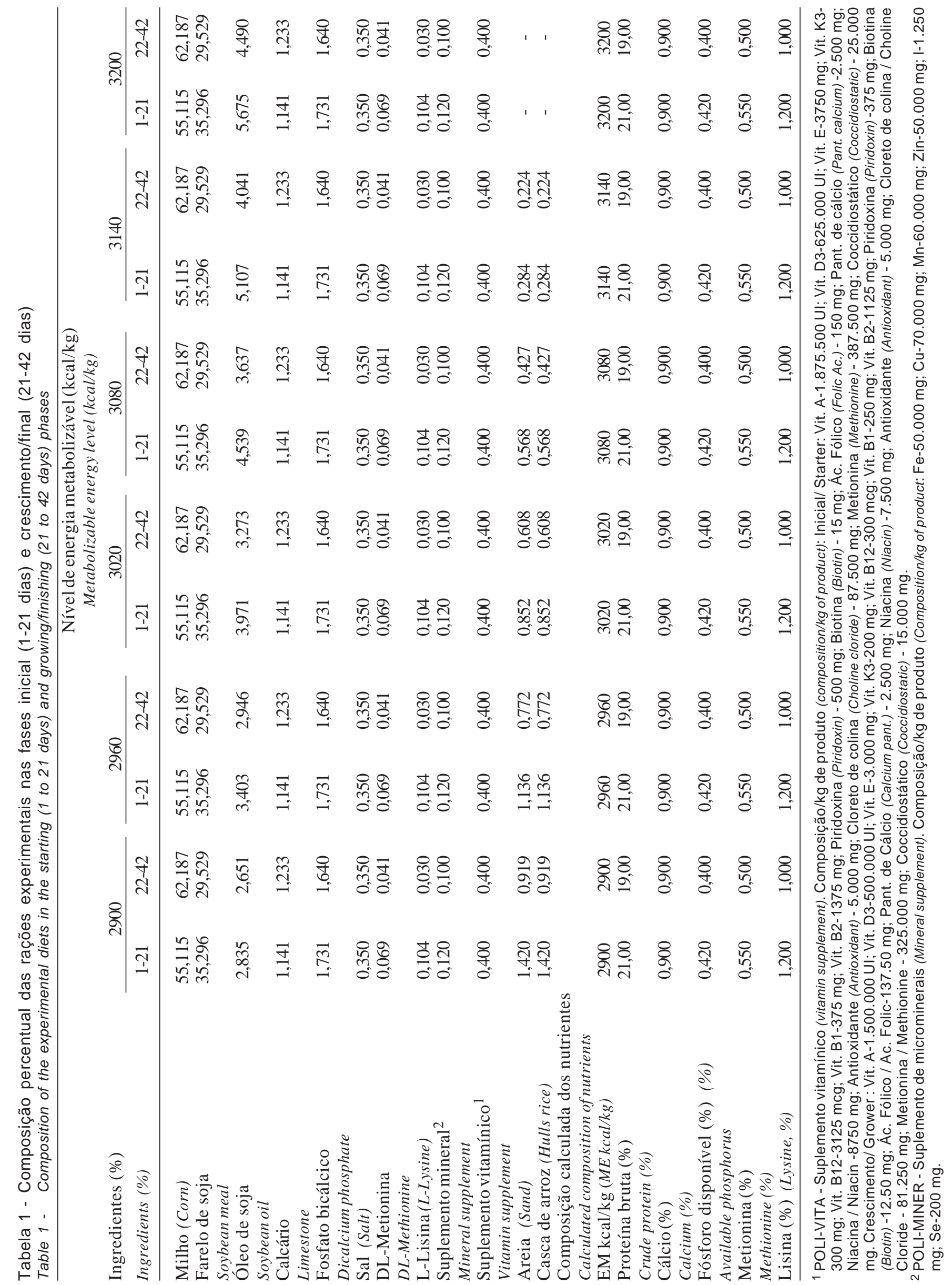


demostrando que o aumento do nível de energia na dieta é a causa do menor consumo de alimento. Embora tenha havido melhora na conversão alimentar, que apresentou efeito cúbico $(p<0,01)$, esta redução não deve ser muito alta, pois pode comprometer o desempenho das aves ao limitar a ingestão de outros nutrientes, como aminoácidos e proteína da dieta. Este fato é evidenciado pelo efeito linear da conversão alimentar até o nível de 3140 kcal $\mathrm{EM} / \mathrm{kg}$, piorando a partir daí.

Os resultados na fase de 1 a 21 dias corroboram os achados de Vasconcelos \& Santos (1997) e Nascimento et al. (1998), que também verificaram avanços no ganho de peso e na conversão alimentar com o aumento do nível de energia da dieta, porém, não encontraram diferenças para o consumo de ração e não relataram os efeitos de níveis altos nesta fase. Porém, Maiorka et al. (1997a), também avaliando o efeito de diferentes níveis de energia na dieta de frangos de corte, não observaram diferenças sobre as variáveis de desempenho no período de 7 a 21 dias, apesar de terem verificado efeito no período de 1 a 7 dias.

No período de 21 a 42 dias de idade, o sexo das aves apresentou o mesmo efeito que na fase inicial. O aumento dos níveis de energia da dieta afetou o consumo de ração e a conversão alimentar, que apresentaram efeitos cúbico $(\mathrm{p}<0,05)$ e linear negativo $(\mathrm{p}<0,05)$, respectivamente, com o aumento do nível de energia da dieta. Houve incremento no consumo de ração com o aumento do nível de energia até $2.960 \mathrm{kcal} \mathrm{EM} / \mathrm{kg}$, de modo que, acima deste nível, a elevação da energia promoveu redução no consumo, porém, com melhora sobre a conversão alimentar, sem afetar, no entanto, o peso vivo e o ganho de peso. Isto comprova que o efeito do nível de energia da dieta sobre o crescimento da ave é diferenciado segundo a fase de criação. Neste trabalho, o uso das mesmas aves da fase anterior ( 1 a 21 dias) pode ter afetado o desempenho na fase de crescimento/ terminação (21 a 42 dias), pois houve comprometimento do desempenho das aves alimentadas com o nível de $3200 \mathrm{kcal}$ EM/kg na fase anterior, comportamento que pode ter persistido até o final do experimento. Mesmo assim, verificou-se melhor desempenho das aves com o aumento dos níveis de energia da dieta, corroborando os achados de Maiorka et al. (1997b), Araújo et al. (1998), Silva et al. (2000) e Moreira et al. (2001). Luchesi (2000) também encontrou avanços no peso vivo e redução do consumo de ração com a elevação da energia da dieta, semelhantemente a Rosa et al. (2000) e Silva et al. (2000), que também relataram efeito linear no desempenho das aves, à medida que se elevou o nível de energia da dieta.

No período de 1 a 42 dias, o sexo afetou os parâmetros avaliados, exceto a mortalidade, sendo que os machos apresentaram melhor desempenho que as fêmeas. Os níveis de energia avaliados afetaram o consumo de ração e a conversão alimentar, pois ambos os parâmetros apresentaram efeito cúbico $(\mathrm{p}<0,05)$, melhorando com o aumento da energia da dieta. Apesar de não ter sido verificado efeito significativo ( $\mathrm{p}>005$ ) para peso vivo e ganho de peso, os melhores resultados foram obtidos com os níveis entre 3.020 e $3.140 \mathrm{kcal} \mathrm{EM/kg}$, o que pode ter sido influenciado, contudo, pelas diferenças ocorridas na fase inicial de criação. Porém, os resultados obtidos permitem concluir que a variação dos níveis de energia da dieta, de 2.900 a $3.200 \mathrm{kcal} \mathrm{EM} / \mathrm{kg}$, promove efeitos diferenciados sobre todos os parâmetros de desempenho das aves, sendo o período de 1 a 21 dias a fase mais afetada por estas variações. Estes resultados corroboram os achados de Rosa et al. (2000), Silva et al. (2000) e Watanabe et al. (2001), que verificaram resultados semelhantes ao avaliarem o efeito de níveis de energia da dieta sobre o desempenho de frangos de corte.

Os resultados de rendimento de carcaça e de partes estão apresentados na Tabela 3. Não houve interação entre os fatores para nenhum dos parâmetros avaliados $(\mathrm{p}>0,05)$. O sexo, apesar de não ter afetado o rendimento da carcaça, teve efeito significativo $(p<0,05)$ sobre o rendimento de partes, sendo que os machos apresentaram maior rendimento de pernas (coxa+sobrecoxas) e de carne de pernas, apesar de apresentarem maiores porcentagens de osso de pernas. Obtiveram-se melhores resultados que os machos para rendimento de peito e de carne de peito, nas fêmeas, que também apresentaram maiores porcentagens de pele de peito e pele de pernas.

Os níveis de energia não afetaram o rendimento de carcaça e de cortes, havendo efeito somente para o rendimento de asas, com efeito linear negativo $(\mathrm{p}<0,05)$ (Figura 1). Este resultado, por não demonstrar consistência, uma vez que a literatura não relata efeito do nível de energia da dieta sobre a porcentagem de asas, deve ser melhor investigado.

A porcentagem de gordura abdominal foi afetada pelo sexo e pelo nível de energia da dieta, sendo que os machos apresentaram $19 \%$ a menos de gordura

R. Bras. Zootec., v.33, n.6, p.2300-2307, 2004 (Supl. 3) 
Tabela 2 - Efeito do nível de energia da dieta e do sexo sobre o desempenho de frangos de corte criados até 42 dias de idade

Table 2 - Effect of dietary energy level and sex on parameters of performance broilers chickens reared until 42 days of age

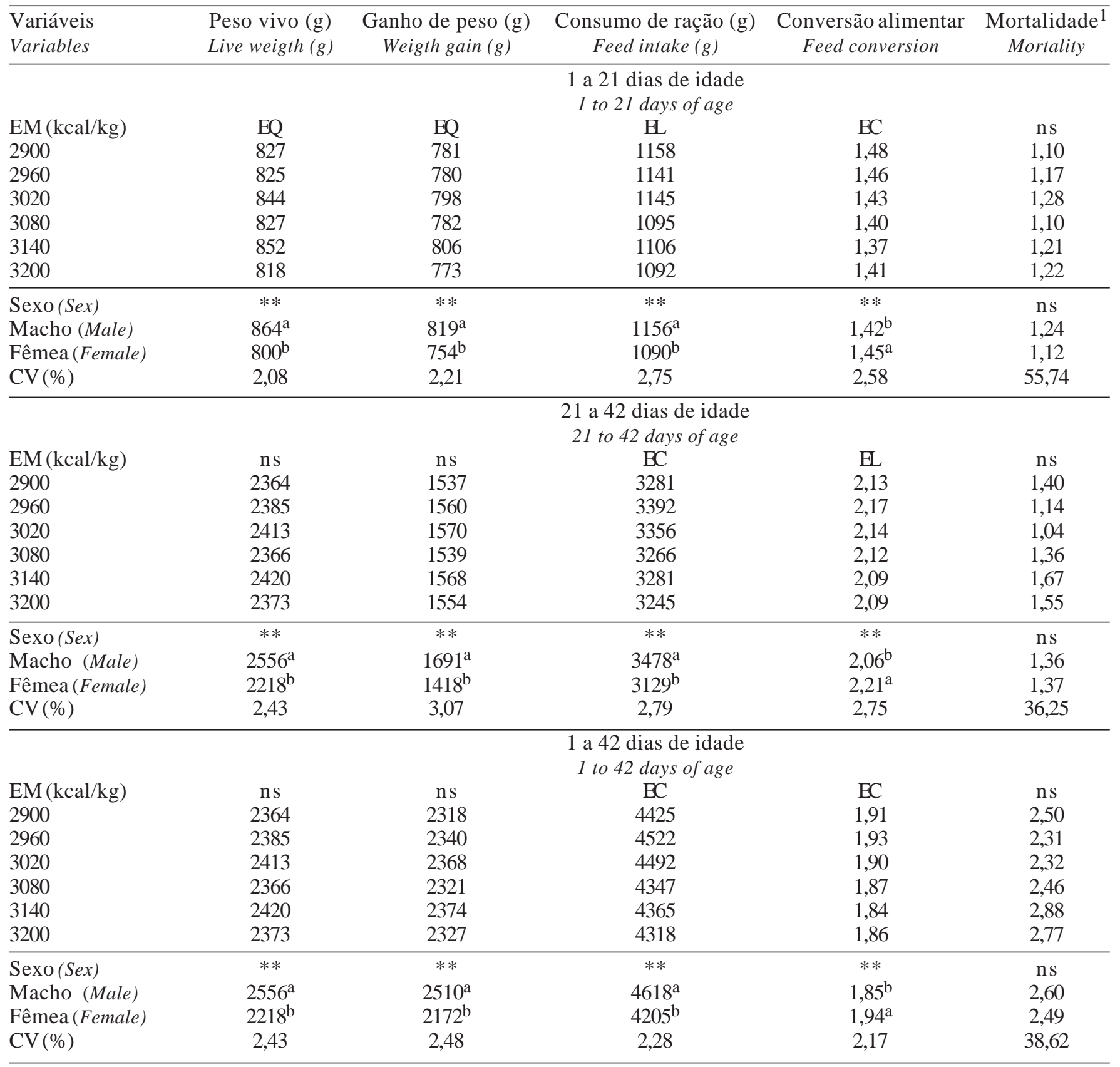

Médias seguidas de letras minúsculas nas colunas diferem entre si pelo teste de Tukey $(p<0,05)$.

Means followed by small letters in column, differ by Tukey test $(p<.05)$.

1 - O percentual de mortalidade foi transformado para raiz (Percentage of mortality was transformed to square/ (mortalidade/mortality (\%) + 0.5) antes da ANOVA/before ANOVA.

EL- Efeito linear $(\mathrm{P}<0,01)$ (Linear effect, $p<.01)$; EQ - Efeito quadrático (Quadractic effect, $p<.01$ ); EC - Efeito cúbico (Cubic effect, $p<.01)$. ns- não-significativo (Not significant); ** significativo (Significant, $p<.01$ ).

que as fêmeas. Os níveis de energia proporcionaram efeito linear $(\mathrm{p}<0,05)$ para gordura abdominal (Figura 2), verificando-se acréscimo da porcentagem de gordura abdominal com o aumento do nível de energia da dieta. De fato, o excesso de energia na dieta é armazenado na forma de gordura.

\footnotetext{
R. Bras. Zootec., v.33, n.6, p.2300-2307, 2004 (Supl. 3)
}

Os resultados de rendimento de carcaça apresentados neste trabalho corroboram os achados de Zanusso et al. (1999), Oliveira Neto et al. (2000) e Leandro et al. (2000), que também não encontraram efeito da energia da dieta sobre o rendimento de carcaça, mas divergem dos relatos 
de Bastos et al. (1998), Rosa et al. (2000) e Mendes et al. (2001), que verificaram resultados diferentes ao avaliarem o efeito de níveis de energia na dieta sobre o rendimento de carcaça. Estes contrastes se justificam pelas diferenças entre linhagens avaliadas, níveis utilizados e balanceamento diferenciado das dietas experimentais. Bastos et al. (1998) encontraram efeitos linear $(\mathrm{p}<0,05)$ e quadrático $(\mathrm{p}<0,05)$ para rendimentos de coxas+sobrecoxas e de peito, respectivamente, o que não foi verificado neste trabalho. Apesar de os níveis de energia avaliados não terem afetado o rendimento de cortes nobres (peito e pernas), há relatos na literatura do efeito da energia da dieta sobre estes parâmetros.

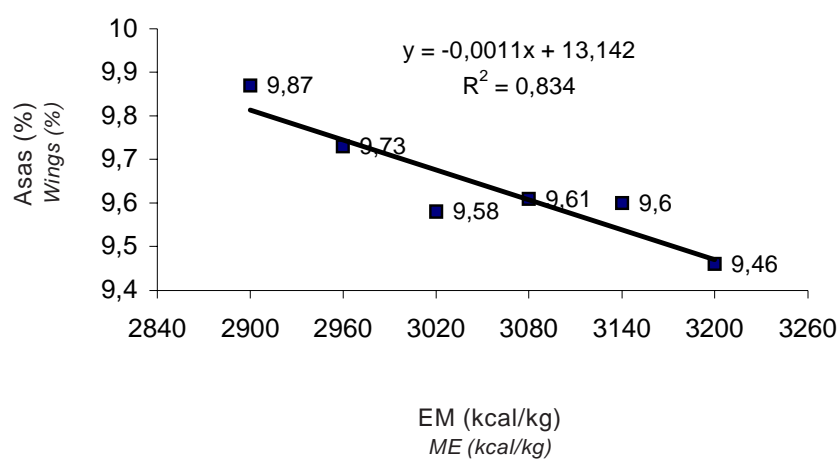

Figura 1 - Efeito do nível de energia da dieta sobre a percentagem de asas em frangos de corte abatidos aos 42 dias de idade.

Figure 1 - Effect of dietary energy level on percentage of wings in broiler chickens slaughtered at 42 days of age.

Tabela 3 - Efeito do nível de energia da dieta e do sexo sobre o rendimento de carcaça e de partes de frangos de corte aos 42 dias de idade

Table 3 - Effect of dietary energy level and sex on carcass yield and parts of broilers at 42 days of age

\begin{tabular}{|c|c|c|c|c|c|c|c|c|c|}
\hline \multirow{2}{*}{$\begin{array}{l}\text { Variáveis } \\
\text { Variables } \\
\text { Rendimento de carcaça e de partes } \\
\text { Carcass yield and parts }\end{array}$} & \multicolumn{6}{|c|}{$\begin{array}{l}\text { Níveis de energia }(\mathrm{EM} \mathrm{kcal} / \mathrm{kg}) \\
\text { Energy level }(\mathrm{ME} \mathrm{kcal} / \mathrm{kg})\end{array}$} & \multicolumn{2}{|c|}{$\begin{array}{l}\text { Sexo } \\
\text { Sex }\end{array}$} & \multirow[b]{2}{*}{ CV,\% } \\
\hline & 2.900 & 2.960 & 3.020 & 3.080 & 3.140 & 3.200 & $\begin{array}{l}\text { Macho } \\
\text { Male }\end{array}$ & $\begin{array}{l}\text { Fêmea } \\
\text { Female }\end{array}$ & \\
\hline $\begin{array}{l}\text { Peso vivo, } g \\
\text { Live weigth, } g\end{array}$ & 2277 & 2402 & 2390 & 2373 & 2402 & 2372 & $2591^{\mathrm{a}}$ & $2147^{b}$ & 4,36 \\
\hline $\begin{array}{l}\text { Carcaça, \% } \\
\text { Carcass \% }\end{array}$ & 82,67 & 81,70 & 82,14 & 83,21 & 82,51 & 82,08 & 82,51 & 82,26 & 1,57 \\
\hline $\begin{array}{l}\text { Asas, }{ }^{1} \\
\text { Wings } \%\end{array}$ & 9,87 & 9,73 & 9,58 & 9,61 & 9,60 & 9,46 & 9,62 & 9,66 & 2,34 \\
\hline $\begin{array}{l}\text { Dorso, \% } \\
\text { Back \% }\end{array}$ & 18,64 & 18,30 & 18,15 & 18,70 & 18,48 & 18,77 & 18,53 & 18,48 & 2,82 \\
\hline $\begin{array}{l}\text { Gordura abdominal, } \%^{1} \\
\text { Abdominal fat \% } \\
\text { Rendimento de peito } \\
\text { Breast yield }\end{array}$ & 2,73 & 2,78 & 2,86 & 2,99 & 2,90 & 3,38 & $2,63^{b}$ & $3,25^{a}$ & 8,44 \\
\hline $\begin{array}{l}\text { Peito, \% } \\
\text { Breast \% }\end{array}$ & 27,03 & 27,78 & 27,11 & 26,87 & 26,96 & 26,79 & $26,50^{\mathrm{b}}$ & $27,68^{\mathrm{a}}$ & 2,56 \\
\hline $\begin{array}{l}\text { Carne peito, \% } \\
\text { Breast meat \% }\end{array}$ & 20,74 & 21,34 & 20,92 & 20,58 & 20,56 & 20,61 & $20,33^{b}$ & $21,25^{\mathrm{a}}$ & 2,96 \\
\hline $\begin{array}{l}\text { Osso peito, \% } \\
\text { Breast bone \% }\end{array}$ & 4,01 & 4,24 & 4,10 & 4,06 & 4,06 & 4,07 & 4,07 & 4,11 & 5,54 \\
\hline $\begin{array}{l}\text { Pele peito, \% } \\
\text { Breast skin \% } \\
\text { Rendimento de pernas } \\
\text { Leg quarter yield }\end{array}$ & 2,28 & 2,20 & 2,09 & 2,23 & 2,34 & 2,11 & $2,10^{\mathrm{b}}$ & $2,32^{\mathrm{a}}$ & 11,82 \\
\hline $\begin{array}{l}\text { Pernas, \% } \\
\text { Legs \% }\end{array}$ & 27,44 & 27,54 & 28,06 & 27,69 & 27,66 & 27,52 & $28,16^{\mathrm{a}}$ & $27,14^{\mathrm{b}}$ & 1,88 \\
\hline $\begin{array}{l}\text { Carne pernas, } \% \\
\text { Leg quarter meat \% }\end{array}$ & 17,80 & 17,54 & 17,84 & 17,63 & 17,60 & 17,56 & $18,12^{\mathrm{a}}$ & $17,18^{b}$ & 3,06 \\
\hline $\begin{array}{l}\text { Osso pernas, \% } \\
\text { Leg quarter bone \% }\end{array}$ & 5,88 & 6,20 & 6,26 & 6,30 & 6,10 & 6,02 & $6,38^{\mathrm{a}}$ & $5,88^{b}$ & 4,99 \\
\hline $\begin{array}{l}\text { Pele pernas, \% } \\
\text { Leg quarter skin \% }\end{array}$ & 3,76 & 3,80 & 3,96 & 3,76 & 3,96 & 3,94 & $3,66^{\mathrm{b}}$ & $4,08^{\mathrm{a}}$ & 9,67 \\
\hline
\end{tabular}

Médias seguidas de letras minúsculas nas linhas, dentro de cada fator, diferem entre si pelo teste de Tukey $(p<0,05)$.

${ }^{1}$ Efeito linear $(p<0,05)$ (Linear effect $[p<.05]$ ).

Means followed by letters small in row, for each factor, differ by Tukey test $(p<.05)$.

R. Bras. Zootec., v.33, n.6, p.2300-2307, 2004 (Supl. 3) 


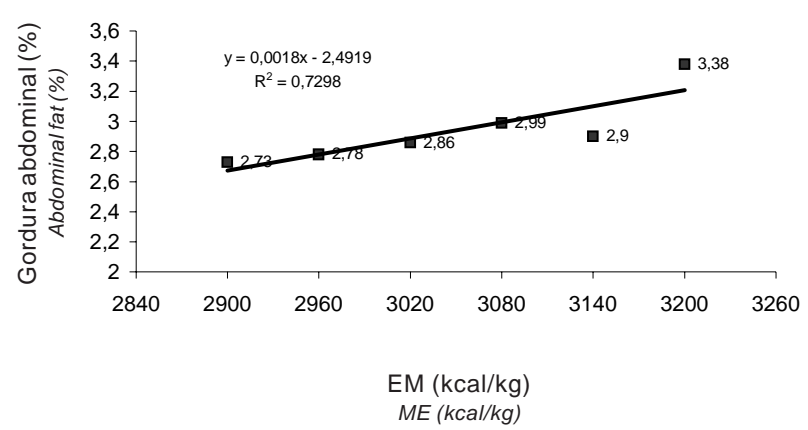

Figura 2 - Efeito do nível de energia da dieta sobre a percentagem de gordura abdominal em frangos de corte abatidos aos 42 dias de idade

Figure 2 - Effect of dietary energy level on abdominal fat percentage in broiler chickens slaughtered at 42 days of age.

\section{Conclusões}

Os machos apresentaram maiores porcentagens de pernas e de carne de pernas e menores de carne de peito e de gordura abdominal que as fêmeas.

\section{Literatura Citada}

ALBUQUERQUE, R.; FARIA, D.E.; JUNQUEIRA O.M. et al. Desempenho e perfil de produção de frangos de corte alimentados com dois níveis de energia na fase final e abatidos em três idades diferentes. In: REUNIÃO ANUAL DA SOCIEDADE BRASILEIRA DE ZOOTECNIA, 2000, 37. Viçosa, MG. Anais... Viçosa, MG: Sociedade Brasileira de Zootecnia, 2000. p.262.

ARAÚJO, L.F.; JUNQUEIRA O.M.; SILVA, C.S. et al. Níveis de energia e lisina para frangos de corte na fase final. In: CONFERÊNCIA APINCO DE CIÊNCIA E TECNOLOGIA AVÍCOLAS, 1998, Campinas. Anais... Campinas: Fundação APINCO de Ciência e Tecnologia Avícola, 1998. p.13.

BASTOS, E.C.G.; LANA, G.R.Q.; SILVA JR., R.G.C. Efeitos de níveis de energia da dieta e do sexo sobre o desempenho produtivo e rendimento de cortes nobres em frangos de corte. In: REUNIÃO ANUAL DA SOCIEDADE BRASILEIRA DE ZOOTECNIA, 35., 1998, Botucatu. Anais... Botucatu: Sociedade Brasileira de Zootecnia, 1998. v.4, p.466-68.

EUCLIDES R.F. Sistema para Análises Estatísticas e Genética - SAEG. Viçosa, MG: Universidade Federal de Viçosa, 1983. 62p.
GIACHETTO P.F.; FERRO J.A.; MACARI M. Influência da restrição alimentar e do nível energético da ração sobre o ganho compensatório em frangos de corte. In: REUNIÃO ANUAL DA SOCIEDADE BRASILEIRA DE ZOOTECNIA, 35., 1998, Botucatu. Anais... Botucatu: Sociedade Brasileira de Zootecnia, 1998. v.4, p.276-78.

LEANDRO N.S.M.; CAFÉ M.B.; MOURA K.A. et al. Influência de diferentes programas alimentares no rendimento de carcaça de frangos de corte. In: REUNIÃO ANUAL DA SOCIEDADE BRASILEIRA DE ZOOTECNIA, 37., 2000, Viçosa, MG. Anais... Viçosa, MG: Sociedade Brasileira de Zootecnia, 2000. p.1149.

LUCHESI, J.B. Nutrição de frangos de corte. In: CONFERÊNCIA APINCO DE CIÊNCIA E TECNOLOGIA AVÍCOLAS, 2000, Campinas. Anais... Campinas: Fundação APINCO de Ciência e Tecnologia Avícola, 2000. v.1, p.111-33.

MAIORKA, A.; LECZNIESKI J.; BARTELS H.A. et al. Efeito do nível energético da ração sobre o desempenho de frangos de corte de 17,7 a 14 e 14 a 21 dias de idade. In: CONFERÊNCIA APINCO DE CIÊNCIA E TECNOLOGIA AVÍCOLAS, 1997, Campinas. Anais... Campinas: Fundação APINCO de Ciência e Tecnologia Avícola, 1997a. p.18.

MAIORKA, A.; KOLLING, A.V.; KESSLER, A.M. et al. Efeito do nível de energia e da forma física da ração em dietas formuladas com base em aminoácidos totais e digestíveis sobre o desempenho e a composição de carcaça de frangos de corte, machos dos 21 aos 42 dias de idade. In: CONFERÊNCIA APINCO DE CIÊNCIA E TECNOLOGIA AVÍCOLAS, 1997, Campinas. Anais... Campinas: FACTA, 1997b. p.19.

MENDES A.A.; MOREIRA J.; GARCIA R.G. et al. Avaliação do rendimento e qualidade da carne de peito em frangos de corte criados com diferentes densidades e níveis de energia na dieta. Revista Brasileira de Ciência Avícolas, p.38, 2001. (Suplemento 3)

MOREIRA, J.; MENDES, A.A.; GARCIA, R.G. et al. Efeito da densidade de criação e do nível de energia da dieta sobre o desempenho e rendimento de carcaça em frangos de corte. Revista Brasileira de Ciência Avícolas, p.39, 2001. (Suplemento 3)

NASCIMENTO, A.H.; ALBINO, L.F.T.; POZZA, P.C. et al. Energia e relação energia:proteína na fase inicial de frangos de corte. In: CONFERÊNCIA APINCO DE CIÊNCIA E TECNOLOGIA AVÍCOLAS, 1998, Campinas. Anais... Campinas: FACTA, 1998. p.15.

OLIVEIRA NETO, A.R.; OLIVEIRA, R.F.M.; DONZELE, J.L. et al. Efeito da temperatura ambiente sobre o desempenho e as características de carcaça de frangos de corte alimentados com dieta controlada e dois níveis de energia metabolizável. Revista Brasileira de Zootecnia, v.29, n.1, p.183-190, 2000.

ROSA, A.P.; BORIN Jr., H.; THIER J. et al. Desempenho e composição de carcaça de frangos submetidos a dietas com diferentes teores energéticos e níveis de gordura. In: REUNIÃO ANUAL DA SOCIEDADE BRASILEIRA DE ZOOTECNIA, 37., 2000, Viçosa, MG. Anais... Viçosa, MG: Sociedade Brasileira de Zootecnia, 2000. p.228.

SILVA J.H.; ALBINO L.F.T.; NASCIMENTO A.H. Energia e relações energia:proteína para frangos de corte de 22 a 42 dias de idade. In: REUNIÃO ANUAL DA SOCIEDADE BRASILEIRA DE ZOOTECNIA, 37., 2000, Viçosa, MG. Anais... Viçosa, MG: Sociedade Brasileira de Zootecnia, 2000. p.264. 
VASCONCELOS, R.Q.; SANTOS M.W.Efeito de níveis de energia e proteína da dieta sobre o desempenho de frangos de corte na fase inicial. In: REUNIÃO ANUAL DA SOCIEDADE BRASILEIRA DE ZOOTECNIA, 34., 1997, Juiz de Fora. Anais... Juiz de Fora: Sociedade Brasileira de Zootecnia, 1997, v.4, p.6-8.

ZANUSSO J.T.; OLIVEIRA, R.F.M.; DONZELE, J.L. et al. Níveis de energia metabolizável para frangos de corte de 1 a 21 dias de idade mantidos em ambiente de conforto térmico. Revista Brasileira de Zootecnia, v.28, n.5, p.1064-1074, 1999.
WATANABE, K.; SAKOMURA, N.K.; RABELLO, C.B.V. et al. Efeito do nível de energia metabolizável da dieta sobre o metabolismo energético de frangos de corte. In: REUNIÃO ANUAL DA SOCIEDADE BRASILEIRA DE ZOOTECNIA, 38., 2001, Piracicaba. Anais... Piracicaba: Sociedade Brasileira de Zootecnia, 2001. p.762.

Recebido em: 11/06/02

Aceito em: 12/04/04 